\title{
Estimating motor unit numbers from a CMAP scan
}

\begin{tabular}{|r|l|}
\hline Journal: & Muscle and Nerve \\
\hline Manuscript ID & MUS-15-0494.R2 \\
\hline Wiley - Manuscript type: & Research Article \\
\hline Date Submitted by the Author: & n/a \\
\hline Complete List of Authors: & $\begin{array}{l}\text { Bostock, Hugh; National Hospital for Neurology and Neurosurgery, MRC } \\
\text { Centre for Neuromuscular Diseases }\end{array}$ \\
\hline Keywords: & CMAP, scan, MUNE, muscle, motor unit potential \\
\hline &
\end{tabular}

SCHOLARONE $^{m}$

Manuscripts 


\title{
Estimating motor unit numbers from a CMAP scan
}

\author{
Hugh Bostock, $\mathrm{PhD}^{1,2}$. \\ ${ }^{1}$ MRC Centre for Neuromuscular Diseases, National Hospital for Neurology and \\ Neurosurgery, Queen Square, London WC1N 3BG, UK \\ ${ }^{2}$ Institute of Neurology, University College London, Queen Square, London WC1N 3BG, UK
}

Running title: CMAP scan MUNE

\section{Corresponding author:}

Professor Hugh Bostock, email: $\underline{\text { H.Bostock@ion.ucl.ac.uk }}$

Acknowledgements: The author acknowledges help from the following, who provided normal CMAP scan data on which the simulated scans in this paper were based, and who also provided useful comments on an earlier version of the manuscript: Laura Nastasi, Department of Clinical Neurophysiology, National Hospital for Neurology and Neurosurgery, London; Hatice Tankisi, Department of Clinical Neurophysiology, Aarhus University Hospital; Peter Grafe, Institute of Physiology, University of Munich; Said Farschtschi, Department of Neurology, University Medical Centre, Hamburg, and James Howells, Brain and Mind Research Institute, University of Sydney, Sydney.

Financial disclosure / Conflict of interest: H.B receives royalties from UCL for sale of the Qtrac software used in this study.

\author{
Word counts: Abstract: 150 words $(\max =150)$ \\ Text: $\quad 3463$ words
}




\begin{abstract}
Introduction: CMAP (compound muscle action potential) scans are detailed stimulus-response curves which provide information about motor unit properties in neuromuscular disorders. This study assessed a method of automatic motor unit number estimation (MUNE) from 5-minute CMAP scans.
\end{abstract}

Methods: A preliminary model, derived from the variance and slope of the scan, is refined to fit the CMAP scan more closely. The method was tested by application to 60 simulated scans, generated from between 5 and 160 motor unit potentials.

Results: The fitting procedure took an average of 1.5 minutes on a standard personal computer. Small unit numbers (5-20) were on average correctly estimated, but large unit numbers $(>40)$ were slightly underestimated. Overall, the absolute MUNE error averaged 6.9\%.

Discussion: This new MUNE method takes all excitable motor units into account and provides realistic estimates of unit numbers over the range 5 to 160 . Validation as a clinical tool awaits further study.

Key words: CMAP, scan, motor unit potential, MUNE, muscle

John Wiley \&²Sons, Inc. 


\section{INTRODUCTION}

If the stimulus to a motor nerve is increased gradually from subthreshold to supramaximal levels, the peak amplitudes of the compound muscle action potential (CMAP) form a detailed stimulusresponse (SR) curve due to the progressive recruitment of more and more motor unit potentials. Such an SR curve has been called a 'muscle scan', or 'CMAP scan' $^{\text {2,3 }}$. It has been used to provide a visual indication of the numbers and sizes of motor units in peripheral neuropathies or motor neuronopathies such as amyotrophic lateral sclerosis (ALS). For many purposes it would be desirable to obtain more specific quantitative information about the motor unit distribution, and in particular a motor unit number estimate (MUNE), for which numerous methods have been proposed since the original incremental method of McComas and colleagues ${ }^{4}$. Of all the MUNE methods described in a recent review ${ }^{5}$, only 1 attempts to derive the detailed distribution of motor unit potentials underlying the CMAP scan. That is the Bayesian statistical method, a highly sophisticated mathematical approach which takes fully into account the probabilistic nature of motor unit firing, but requires several hours of intensive computation to come up with the most probable set of units that could have generated a scan ${ }^{6,7}$. The aim of this study was to test an alternative, more rough-and-ready method, designed similarly to derive the population of units underlying a scan, but in minutes rather than hours. The essence of the new method is that a recorded scan is compared to a modelled scan by converting the scans to contour maps and then using the degree of overlap of the contours to assess the accuracy of the model. Since it is only in cases of severe denervation that the true number of functional motor units in a muscle is known, the method has been tested on simulated CMAP scans, similar to those used to illustrate the muscle scan method ${ }^{1}$.

John Wiley \& $^{3}$ Sons, Inc. 


\section{METHODS}

There were 5 distinct methods used in this study. These were: (1) the method used to record a CMAP scan from a patient or normal subject; (2) the mathematical model used to simulate the CMAP scans; (3) the method of scoring how close a simulated scan is to the recorded one; (4) the method used to fit a model to a recorded scan; and (5) the method used to test how well the fitting procedure works. Methods 2-4 are contained within the MScanFit program, initially written as part of the author's QTRACW software (C Institute of Neurology, University College London, distributed by Digitimer Ltd at www.digitimer.com). A separate, stand-alone version of MScanFit (i.e. MScanFit.exe) is now available as freeware from the author (․ㅡ.Bostock@ucl.ac.uk). It differs from the QTRACW version in taking CMAP scan data from a simple text file, rather from a QTRACW MEM file.

\section{(1) Method of recording CMAP scan}

CMAP scans are available as part of the TRONDNF recording protocol in the QTRACW software (see above). The CMAP scans used in this paper were all recorded by stimulating the median nerve at the wrist and recording with the active electrode over the motor point of abductor pollicis brevis, and the reference electrode over the proximal phalanx. After manually setting the stimulus to a just supramaximal level, a pre-scan set of 20 CMAPs is recorded, then the stimulus is decreased in regular percentage steps (usually $0.2 \%$ ) until the response disappears. A further 20 post-scan sweeps are then recorded (Figure 1). The pre- and post-scan sweeps are used to assess the variability of the responses and the baseline noise. The CMAP amplitudes are measured as baseline-to-peak (see Fig 1C). To mitigate the effects of baseline noise, the baseline is set at the average of 10 sequential points in the trace during the period between the end of the stimulus artifact and start of the CMAP. An exponential rather than linear

John Wiley \&4 Sons, Inc. 
decline in stimulus amplitude is used, since motor axon threshold variability is proportional to threshold, and the exponential decline provides similar numbers of stimuli to assess the thresholds of low threshold units as of high threshold units. In normal nerves, stimulus strengths from first detectable response to maximal CMAP amplitude typically vary by a factor of 2 to 2.5 (e.g. from 8 to $20 \mathrm{~mA}$ in Figure 1A), so that they require about 350 to $4500.2 \%$ steps to cover the range. With the pre-scan and post-scan sweeps this takes about 3.5 to 5 minutes when stimulating at $0.6 \mathrm{~s}$ intervals. The number of stimuli in these exponential scans is variable, but it is similar to the 500 at $2 \mathrm{~Hz}$ previously recommended for linear CMAP scans ${ }^{3}$. Baseline noise and stimulus artifact affect the quality of CMAP scans, thus careful skin preparation and low impedance electrodes are essential for good quality recordings.

\section{(2) Mathematical model used to simulate CMAP scans}

The model consists of $N$ units, each described by 3 parameters: threshold $(t)$, amplitude $(m)$, and relative spread of threshold $(\rho)$, which follows a Gaussian function as previously found for real motor unit potentials ${ }^{8}$. Relative spread is the coefficient of variation of the threshold, expressed as a percentage, i.e. $100 \times(\mathrm{SD}$ of $t) / t$. The $N$ units are assumed to provide a fixed contribution (m) to CMAP amplitude, but 2 additional variables allow for baseline noise and variability in the peak CMAP, so that a model of $N$ motor units is described by a total of $3 N+2$ parameters.

\section{(3) Scoring of discrepancy between model and recorded scan}

MScanFit allows for uncertainty in the thresholds and responses of a single scan by smoothing each point on the scan in $x$ and $y$ directions. This smoothed scan, which represents the probability of a particular stimulus evoking a particular response for all possible stimuli and responses, can be plotted as a contour map. A simulated scan can be generated by applying a matching set of stimuli to the model, and this can also be smoothed to generate a contour map. 
An error score for the discrepancy between the nerve and the model is then calculated by summing the squares of the differences between the contoured values at each stimulus-response point and expressing this as a percentage of the sums of squares of the contoured values of the recorded scan. If the model were completely accurate, the error score would tend to zero as more and more stimuli were delivered to the nerve and the model. The number of stimuli delivered to the nerve is fixed before the analysis, so the contour map of the recorded scan is inevitably rather noisy. However, the number of stimuli delivered to the model can be much greater, so that the contour map of the model scan is much smoother. In MScanFit the number of stimuli delivered to the model is varied between 5 and 20 times the number in the recorded scan, depending on the number of points in the recorded scan and the estimated number of units.

\section{(4) Fitting model to recorded CMAP scan}

The model is fitted to a scan in 3 stages: (4a) The pre-scan and post-scan periods are checked manually to ensure they provide appropriate estimates of CMAP variability at those stages; (4b) A preliminary model is derived from an analysis of the slope and variance of the scan as a function of stimulus intensity (see below); and (4c) The model is refined by making successive adjustments to the parameters of the model until the discrepancy (as defined above) cannot be reduced further (see below). As with other MUNE methods, it was necessary to limit the smallest size for a motor unit potential. A size limit of $25 \mu \mathrm{V}$ has been used for multipoint incremental $\mathrm{MUNE}^{9}$, but in other studies the limit has been set at $40 \mu \mathrm{V}^{10}$ or even as high as 100 $\mu \mathrm{V}^{6}$. The latter figure was used for Bayesian statistical MUNE, although it sets a rather low limit to the number of units that can be estimated for a normal, $10 \mathrm{mV} \mathrm{CMAP.} \mathrm{If} \mathrm{the} \mathrm{size} \mathrm{limit} \mathrm{is}$ set too small, inappropriate extra units may be added by the fitting procedure to reduce the discrepancy, whereas if it is set too large, then genuine motor unit potentials may be missed. In this study the greatest accuracy over the range of simulated scans was obtained by varying the 
limit from $25 \mu \mathrm{V}$, when the average unit size was less than $125 \mu \mathrm{V}$, to $50 \mu \mathrm{V}$ when the average unit size exceeded $250 \mu \mathrm{V}$. The $\rho$ values were always initialized at $2.0 \%$, although the target scans were generated with $\rho=1.65 \%$ (see below), because this was found to result in more accurate MUNE values..

\section{(4b) Generation of preliminary models}

Each unit within the CMAP makes a contribution to the variance of the CMAP and the slope of the stimulus-response curve within the region of stimulus strength that it fires probabilistically. From the ratio between these 2 quantities and the threshold variability, it is possible to estimate the number of units recruited over a range of stimulus strengths (see Appendix, available online). This principle is used to generate preliminary models. It turns out that these models depend critically on the number of data points over which the variance and slope are calculated. MScanFit therefore calculates variance and slope over different numbers of points, generates a preliminary model from each, and then selects the one which generates the lowest error according to method (3) above. In addition to its function of providing a preliminary model from the estimated number of units, this part of the program can also be used to generate a model with any specified number of units that produces a scan with a similar distribution of slopes and variances.

\section{(4c) Refining the model}

To improve the model, MScanFit first calculates a separate error contribution for each stimulus strength applied. The unit in the model with threshold closest to the stimulus at which the greatest error occurs is then selected first, and its properties changed until a change is found that reduces the error score by more than $1 \%$. This change is then made permanent, the errors for each stimulus strength are recalculated, and the process is repeated. When no change in the first

John Wiley \& ${ }^{7}$ Sons, Inc. 
selected unit is effective, the unit with threshold closest to the stimulus at which the next highest error occurs is selected, and its properties are changed as before to reduce the error. This procedure is repeated until no change in any of the first $n$ units tested reduces the error further (where $n$ is between 10 and 60). The size of the changes is then halved, and the whole process is repeated, halved again and the whole process repeated again. The changes in model units tested include increasing and decreasing each of its 3 parameters: threshold, amplitude, and relative spread. Also tested are splitting 1 unit into 2, to increase the number of units, and combining 1 unit with an adjacent unit, to reduce the number of units. Altogether 11 changes are tested for each unit, and after each change, between 5 and 20 simulated scans are generated to compare with the recorded scan according to method (3).

Several such model refinements are carried out, depending on the number of units in the model. If the refining process increases the number of units, then another preliminary model is generated with more units (using method $4 \mathrm{~b}$ ), whereas if the number is reduced, a preliminary model is generated with fewer units, and the process is repeated up to 9 times. Each time the process is repeated, the values of relative spread are reset to the weighted mean of the values from the last refinement. The model which produces the simulated scan with the lowest error score of all the models tested is presumed to provide the best MUNE.

The CMAP scan fitting procedure is illustrated in Figures 2 and 3. The scan to be fitted (which in this case is a simulated scan derived from 20 units) is shown in Figure 2Aa. From the slope and variance of the scan, a preliminary model with 18 units is derived, from which the scan in Figure $2 \mathrm{Ba}$ is generated. The scans in $2 \mathrm{Aa}$ and $2 \mathrm{Ba}$ are compared by smoothing the points in the $x$ and $y$ directions to generate the contour maps in 2Ab and 2Bb. From the difference between these 2 contour maps, illustrated in Figure $3 \mathrm{Ab}$, an error score of 11.0 is calculated. The refinement process is then started, which progressively reduces the difference between the 
contour maps by modifying the properties of the different units until a minimum error score of 0.61 is achieved (Figure $3 \mathrm{Bb}$ ), by which time the number of units has increased to 20 . The refined model generates the scan in Figure $2 \mathrm{Ca}$ and the contour map in Figure $2 \mathrm{Cb}$. The units in the model generating the target scan, and the units in the refined fitted model, each consisting of 20 units with different amplitudes, thresholds, and threshold variability, are illustrated schematically in Figure 3C and 3D respectively. It can be seen that the threshold distributions are very similar, but not identical (e.g. the relative sizes of the 2 biggest units are reversed in 3D).

\section{(5) Testing the accuracy of the fitting procedure}

The MScanFit program was tested by generating simulated CMAP scans from models with defined numbers of units (namely 5, 10, 20, 40, 80, and 160) and then running MScanFit to see how accurately the unit distribution was estimated. The models were generated by first using 10 CMAP scans from normal subjects as templates to generate models with 160 units (see 4b above) each with $\rho$ set to $1.65 \%$, corresponding to the mean value found experimentally ${ }^{8}$. The number of units in each model was then halved in successive stages in such a way as to crudely simulate substantial collateral reinnervation. Twenty percent of the units were selected at random and removed, while another $30 \%$ were selected at random and their amplitudes added to a randomly selected surviving unit. Thus each halving of the number of units produced on average only a $20 \%$ reduction in the maximal CMAP amplitude. Single scans generated by each of the 60 models were then analyzed by MScanFit to estimate the number of units. One such sequence is illustrated in Figure 4. In each of the 6 panels A-F, the left hand scan is the target scan, derived from a model with a defined number of units, and the right hand scan is derived from the fitted model. The scans are displayed in reverse order of generation, since the 160-unit model in F was created first, and the remainder generated by halving the units in order F-E-D-C- 
B-A. It can be seen that the fitted scans look quite similar to the target scans even though the number of units is different. One obvious effect of the simulated collateral reinnervation is that the largest single unit amplitude (LSU) and the mean single unit amplitude (MSU) tend to increase as the number of units decreases. The estimates of these quantities (LSUE, MSUE) as well as the estimated number of motor units (MUNE) were compared with the values in the model that generated the target scan, to provide measures of the accuracy of the fitting procedure.

\section{Subjects}

Ten healthy normal control subjects were used to obtain CMAP scans from which the simulated scans were derived. The subjects comprised 6 men and 4 women, mean age 31.2 years (range 20 -48 years). The procedure was approved by the local ethics committees (The Joint Institute of Neurology/National Hospital for Neurology and Neurosurgery Research Ethics Committee, Danish National Committee on Biomedical Research Ethics in Central Denmark Region, Hamburg University Ethics Committee, Sydney University Human Research Ethics Committee) and conformed to the Declaration of Helsinki. The subjects gave their written informed consent.

\section{RESULTS}

The results of the testing study are summarized in Table 1. The first row shows that the MScanFit program takes less than a minute, on average, to fit scans generated by 5 units, and only 1.5 minutes, on average, to fit all 60 scans. All the scans were fitted within 3 minutes, when MScanFit was run on a personal computer (Intel i5, $2.5 \mathrm{GHz}$, Windows 7). The second row shows that the MUNE value was estimated quite accurately when the true number was small $(<30)$, but slightly underestimated when the number was large $(>30)$. This is shown more clearly

John Wiley \& Sons, Inc. 
by the percentage errors in the third row of the table, and diagrammatically in Figure 5B.

Comparing Figures 5A and 5B, it is evident that the refinement is effective in reducing the MUNE error when the number of units is small, but rather ineffective when the number is large. Probably the best measure of the overall accuracy of the estimates is the mean absolute percentage error (scoring all errors as positive), and these values are shown in the fourth row of Table 1. The last column shows that for the 60 scans the mean absolute percentage MUNE error was less than $7 \%$.

MScanFit provides more information about the motor unit population than just the number of units, and estimates of largest single unit amplitude (LSUE) provide a measure of successful collateral reinnervation. Table 1 shows that this quantity is remarkably accurate for scans with small numbers of motor unit potentials, with the largest of 5 units, averaging more than $1 \mathrm{mV}$, estimated with an average absolute error of less than $5 \mu \mathrm{V}$. When there was extensive overlap, however, the largest motor unit potentials tended to get 'lost in the noise', and the estimates were much less accurate. Nevertheless, the mean absolute LSUE error for the 60 scans was only $40 \mu \mathrm{V}$, which was equal to the amplitude resolution of the contour maps. Interestingly, although the mean single unit amplitudes (MSU) increased proportionately more than LSU as the number of units decreased with collateral reinnervation (from 62.5 to $523 \mu \mathrm{V}$ ), the estimates of this quantity (MSUE) were even more accurate than LSUE, with an average absolute error for the 60 scans of only $11 \mu \mathrm{V}$.

\section{DISCUSSION}

This paper describes a new method of analyzing CMAP scans and shows that for simulated scans with a wide range of unit numbers, MUNE values were derived within an average of 1.5 minutes with a mean absolute error of less than $7 \%$. Additionally, mean single

John Wiley \& Sons, Inc. 
unit amplitudes were estimated with a mean absolute error of only $11 \mu \mathrm{V}$. This method may prove useful in detecting and quantifying motor unit loss in peripheral neuropathies and neuronopathies, but it is important to be aware that the accuracy determined in this study by testing against simulated CMAP scans is most unlikely to be achieved in recordings from real nerves and muscles. Rather, it indicates the maximum accuracy that could be achieved if the real scans behaved like the idealized simulated scans.

Probably the most serious simplification in the modelling is that the contribution to peak CMAP amplitude of each motor unit is assumed to be constant, whereas it can change for a variety of reasons, including progressive changes in latency with stimulus amplitude, activitydependent changes in muscle fiber action potentials, and in the case of ALS and other conditions with unstable collateral reinnervation, because of fluctuations in the number of muscle fibers contributing to the motor unit potential. This additional source of CMAP variability has been held responsible for artifactual increases in statistical MUNE in $\mathrm{ALS}^{10}$. In MScanFit, spontaneous fluctuations in motor unit potential size are taken into account, to some extent, by providing for a progressive change in noise level between the pre-scan and post-scan periods, but no attempt has been made to simulate the effects of unstable motor units on the analysis.

Another simplification in the simulated scans used as targets was that the relative spread of threshold $(\rho)$ was made the same for all units, and equal to the average of $1.65 \%$ found for normal human units ${ }^{8}$. The variability in $\rho$ values found in that study (SD of $0.26 \%$ ) was not simulated, and $\rho$ values in diseases such as ALS have yet to be investigated. Surprisingly, perhaps, it was found that a higher initial value for $\rho$ in the fitted models (2\%) gave more accurate MUNE values when there was a lot of overlap in thresholds. This may have been because the formula used to estimate motor unit potential size from the variance and slope of the scan (equation [3] in Appendix) assumes that the potentials are of equal size. When units are of unequal size, the variance is increased, so that unit size is overestimated and unit number 
underestimated. Increasing $\rho$ compensated for this, however the use of a high initial $\rho$ value did not prevent the model refinement process from accurately estimating the unit numbers when they were low and discrete levels were discernible in the muscle scans.

Despite these limitations on the use of CMAP scans to generate MUNE, the method has advantages over other types of MUNE, which may make it the method of choice in some circumstances. CMAP scan MUNE depends on, and provides information about, all the motor units contributing to the CMAP, whereas incremental (including multipoint) MUNE is determined by the units with lowest electrical thresholds only. Most statistical MUNE methods depend on responses at only a few stimulus levels, and MUNIX and spike-triggered averaging methods depend primarily on units recruited at low levels of voluntary effort. The Bayesian statistical MUNE method $^{6,7}$, like MScanFit, attempts to provide an estimate of all the motor units in a muscle, but it requires complex, off-line computations, available only in a limited number of centers $^{5}$ and can take several hours to complete, even with a high-powered computer. MScanFit is much less mathematically sophisticated, but this study shows that in principle it produces reasonably accurate MUNE values on a laptop in less than 3 minutes. The program is available as freeware to apply to CMAP scan data in simple text files. The CMAP scan is already established as a simple and useful technique ${ }^{1-3}$ and the method described here promises to extend its usefulness by the addition of a credible MUNE value.

John Wiley \& Sons, Inc. 


\begin{abstract}
ABBREVIATIONS
ALS = Amyotrophic lateral sclerosis

CMAP $=$ Compound muscle action potential

LSU(E) $=$ Largest single unit amplitude (estimate)

$\operatorname{MSU}(\mathrm{E})=$ Mean single unit amplitude (estimate)

$\operatorname{MUN}(\mathrm{E})=$ Motor unit number (estimate)
\end{abstract}

John Wiley \& 1 Sons, Inc. 


\section{REFERENCES}

1. Blok JH, Ruitenberg A, Maathuis EM, Visser GH. The eletrophysiological muscle scan. Muscle Nerve 2007;36:436-446.

2. Maathuis EM, Drenthen J, Visser GH, Blok JH. Reproducibility of the CMAP scan. J Electromyogr Kinesiol 2010;21:433-437.

3. Maathuis EM, Henderson RD, Drenthen J, Hutchinson NM, Daube JR, Blok JH, Visser GH. Optimal stimulation settings for CMAP scan registrations. J Brachial Plex Peripher Nerve Inj 2012;7:4.

4. McComas AJ, Fawcett PR, Campbell MJ, Sica RE. Electrophysiological estimation of the number of motor units within a human muscle. J Neurol Neurosurg Psychiatry $1971 ; 34: 121-131$.

5. Gooch CL, Doherty TJ, Chan KM, Bromberg MB, Lewis RA, Stashuk DW et al. Motor unit number estimation: a technology and literature review. Muscle Nerve 2014;50:884893.

6. Ridall PG, Pettit AN, Henderson RD, McCombe PA. Motor unit number estimation - a Bayesian approach. Biometics 2006;62:1235-1250.

7. Henderson RD, Ridall PG, Hutchinson NM, Pettit AN, McCombe PA. Bayesian statistical MUNE method. Muscle Nerve 2007;36:206-213.

8. Hales JP, Lin CS-Y, Bostock H. Variations in excitability of single human motor axons, related to stochastic properties of nodal sodium channels. J Physiol 2004;559:953-964.

John Wiley \&.5ons, Inc. 
9. Shefner JM, Watson MI, Simionescu L, Caress JB, Burns TM, Maragkis NJ et al. Multipoint incremental motor unit number estimation as an outcome measure in ALS. Neurology 2011;77:235-241.

10. Shefner JM, Cudkowicz ME, Zhang MA, Schoenfeld D, Jillapalli D, Northeast ALS Consortium. The use of statistical MUNE in a multicenter clinical trial. Muscle Nerve 2004;30:463-469.

John Wiley \& Sons, Inc. 


\section{FIGURE LEGENDS}

Figure 1. Example of recording of CMAP scan from abductor pollicis brevis of a normal subject, stimulating the median nerve at the wrist. (A) Stimulus level, which started supramaximal, and after a pre-scan series of 20 CMAPs, was decreased in $0.2 \%$ steps, which continued for 20 post-scan stimuli after the response amplitude had fallen to zero (stimulus duration $0.2 \mathrm{~ms}$ ). B: Peak CMAP amplitudes. C: 470 superimposed CMAP waveforms that made up the CMAP scan.

Figure 2. CMAP scans for model with 20 units, showing fitting procedure. Aa: The target scan, which is the input to the MScanFit program. Ba: CMAP scan generated by preliminary model with 18 units, based on slopes and variances of data in Aa. Ca: CMAP scan generated by optimized model with 20 units. Ab, Bb, Cb: contour maps obtained by smoothing CMAP scans in $x$ and $y$ directions, which were used to assess discrepancy between scans. The percentage error between preliminary scan $\mathrm{Bb}$ and target scan $\mathrm{Ab}$ was 11.0, whereas between optimized scan $\mathrm{Cb}$ and $\mathrm{Ab}$ it was 0.61 (see also Figure 3).

Figure 3. Contour map differences used to compare CMAP scans. Ab: Difference between contour maps $\mathrm{Ab}-\mathrm{Bb}$ in Figure 2, where red lines indicate $\mathrm{Ab}>\mathrm{Bb}$, and green lines indicate $\mathrm{Ab}$ $<\mathrm{Bb}$. Bb: Difference contour Ab-Cb in Figure 2. Aa, Ba: Peak differences at each level of stimulus current. C: The target 20 motor unit distribution used to generate CMAP scan Aa in Figure 2. Peak height indicates unit amplitude, and a Gaussian distribution indicates threshold probability. D: Similar plot for optimized model. 
Figure 4. Example of a sequence of target scans, with defined numbers of units from 5 to 160 (left half of each panel), and scans generated by models fitted by MScanFit, indicating motor unit number estimates (right half of each panel). The target scans were derived from the 160unit model in F, by progressive halving of the numbers of units. Note that Figure 4A provides an interesting example of alternation: 3 units had overlapping thresholds between 25 and $30 \mathrm{~mA}$, giving rise to multiple amplitude levels, which were successfully interpreted by the program.

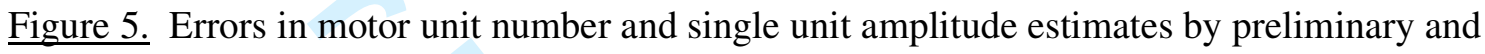
refined models of MScanFit. A,B: MUNE errors, expressed as percentages. C,D: MSUE errors in $\mu \mathrm{V}$. A,C: Values from preliminary model. B,D: Values from refined model. In each case, the box and whisker plots indicate the median, interquartile range, and total range of the errors in the 10 estimated values. Note that for 5 units in B, there is no box or whisker visible, since all estimates had zero error.

John Wiley \& \&ons, Inc. 


\begin{tabular}{|c|c|c|c|c|c|c|c|}
\hline MScanFit results & $\begin{array}{c}5 \\
n=10\end{array}$ & $\begin{array}{c}10 \\
n=10\end{array}$ & $\begin{array}{c}20 \\
n=10\end{array}$ & $\begin{array}{c}40 \\
n=10\end{array}$ & $\begin{array}{c}80 \\
n=10\end{array}$ & $\begin{array}{c}160 \\
n=10\end{array}$ & $\begin{array}{l}\text { Combined } \\
n=60\end{array}$ \\
\hline Time to fit (min) & $\begin{array}{c}0.8 \\
\pm 0.2\end{array}$ & $\begin{array}{c}1.6 \\
\pm 0.4\end{array}$ & $\begin{array}{r}1.8 \\
\pm 0.5\end{array}$ & $\begin{array}{c}1.7 \\
\pm 0.3\end{array}$ & $\begin{array}{c}1.3 \\
\pm 0.3\end{array}$ & $\begin{aligned} & 1.8 \\
\pm & 0.5\end{aligned}$ & $\begin{array}{r}1.5 \\
\pm 0.5\end{array}$ \\
\hline MUNE & $\begin{array}{l}5.0 \\
\pm 0.0\end{array}$ & $\begin{array}{r}10.1 \\
\pm 0.6\end{array}$ & $\begin{array}{r}19.6 \\
\pm 2.0\end{array}$ & $\begin{array}{r}38.3 \\
\pm 3.9\end{array}$ & $\begin{array}{r}73.5 \\
\pm 6.5\end{array}$ & $\begin{array}{r}139.7 \\
\pm 19.8\end{array}$ & \\
\hline MUNE (\% error) & $\begin{array}{c}0.0 \\
\pm 0.0\end{array}$ & $\begin{array}{c}1.0 \\
\pm 5.7\end{array}$ & $\begin{array}{r}-2.0 \\
\pm 9.8\end{array}$ & $\begin{array}{r}-4.3 \\
\pm 9.8\end{array}$ & $\begin{array}{l}-8.1 \\
\pm 8.1\end{array}$ & $\begin{array}{l}-12.7 \\
\pm 12.4\end{array}$ & $\begin{array}{r}-4.3 \\
\pm 9.5\end{array}$ \\
\hline MUNE (absolute \% error) & $\begin{array}{c}0.0 \\
\pm 0.0\end{array}$ & $\begin{array}{c}3.0 \\
\pm 4.8\end{array}$ & $\begin{array}{r}8.0 \\
\pm 5.4\end{array}$ & $\begin{array}{r}8.3 \\
\pm 6.4\end{array}$ & $\begin{array}{l}9.1 \\
\pm 6.8\end{array}$ & $\begin{array}{c}13.2 \\
\pm 11.8\end{array}$ & $\begin{array}{c}6.9 \\
\pm 7.8\end{array}$ \\
\hline $\begin{array}{l}\text { Largest single unit (LSU) } \\
\quad \text { in target scan }(\mu \mathrm{V})\end{array}$ & $\begin{array}{r}1238 \\
\pm 100\end{array}$ & $\begin{array}{r}745 \\
\pm 100\end{array}$ & $\begin{array}{r}856 \\
\pm 87\end{array}$ & $\begin{array}{l}851 \\
\pm 94\end{array}$ & $\begin{array}{r}395 \\
\pm 53\end{array}$ & $\begin{array}{l}210 \\
\pm 74\end{array}$ & \\
\hline $\begin{array}{l}\text { LSU estimate (LSUE) } \\
\qquad(\mu \mathrm{V})\end{array}$ & $\begin{array}{l}1236 \\
\pm 98\end{array}$ & $\begin{array}{l}761 \\
\pm 99\end{array}$ & $\begin{array}{r}879 \\
\pm 79\end{array}$ & $\begin{array}{r}875 \\
\pm 99\end{array}$ & $\begin{array}{r}319 \\
\pm 90\end{array}$ & $\begin{array}{l}221 \\
\pm 65\end{array}$ & \\
\hline $\begin{array}{l}\text { LSUE (absolute error, } \\
\qquad \mathrm{V} \text { ) }\end{array}$ & $\begin{array}{c}4 \\
\pm 5\end{array}$ & $\begin{array}{r}16 \\
\pm 7\end{array}$ & $\begin{array}{c}27 \\
\pm 31\end{array}$ & $\begin{array}{c}39 \\
\pm 24\end{array}$ & $\begin{array}{c}83 \\
\pm 85\end{array}$ & $\begin{array}{c}70 \\
\pm 58\end{array}$ & $\begin{array}{c}40 \\
\pm 52\end{array}$ \\
\hline $\begin{array}{l}\text { Mean single unit (MSU) } \\
\text { in target scan }(\mu \mathrm{V})\end{array}$ & $\begin{array}{l}523 \\
\pm 28\end{array}$ & $\begin{array}{l}286 \\
\pm 14\end{array}$ & $\begin{array}{l}254 \\
\pm 7\end{array}$ & $\begin{array}{l}170 \\
\pm 4\end{array}$ & $\begin{array}{l}100 \\
\pm 2\end{array}$ & $\begin{array}{l}62.5 \\
\pm 0\end{array}$ & \\
\hline $\begin{array}{l}\text { MSU estimate (MSUE) } \\
(\mu \mathrm{V})\end{array}$ & $\begin{array}{l}523 \\
\pm 28\end{array}$ & $\begin{array}{r}284 \\
\pm 19\end{array}$ & $\begin{array}{l}261 \\
\pm 25\end{array}$ & $\begin{array}{r}179 \\
\pm 20\end{array}$ & $\begin{array}{r}110 \\
\pm 10\end{array}$ & $\begin{array}{r}73 \\
\pm 11\end{array}$ & \\
\hline $\begin{array}{l}\text { MSUE (absolute error, } \\
\mu \mathrm{V})\end{array}$ & $\begin{array}{c}0.4 \\
\pm 0.8\end{array}$ & $\begin{array}{c}8 \\
\pm 13\end{array}$ & $\begin{array}{c}21 \\
\pm 15\end{array}$ & $\begin{array}{c}16 \\
\pm 14\end{array}$ & $\begin{array}{r}10 \\
\pm 8\end{array}$ & $\begin{array}{c}11 \\
\pm 11\end{array}$ & $\begin{array}{c}11 \\
\pm 13\end{array}$ \\
\hline
\end{tabular}

Table 1. Results of fitting 60 simulated scans with MScanFit. Numbers in each box indicate means \pm standard deviations.

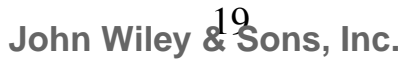



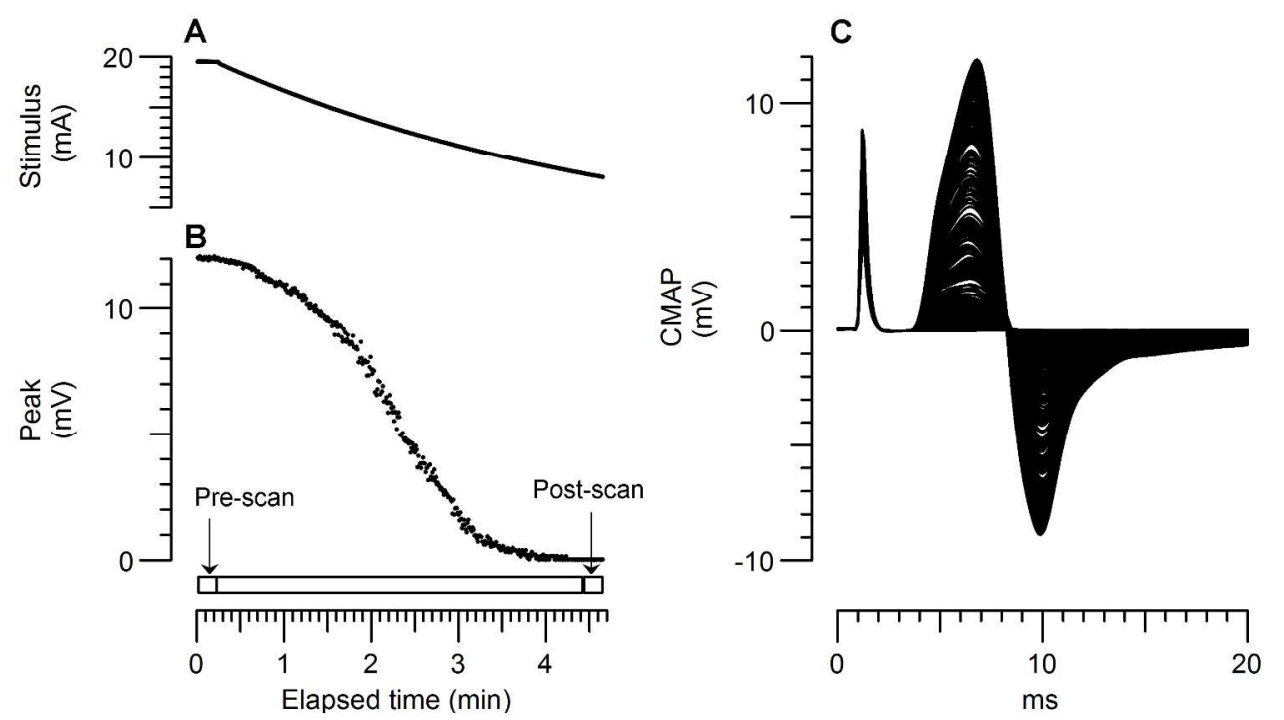

Figure 1

$254 \times 190 \mathrm{~mm}(300 \times 300$ DPI $)$

34

35

36

37

38

39

40

41

42

43

44

45

46

47

48

49

50

51

52

53

54

55

56

57

58

59

60

John Wiley \& Sons, Inc. 


\section{Page 21 of 24}

Figure 2

$254 \times 190 \mathrm{~mm}$ (300 x 300 DPI)

John Wiley \& Sons, Inc. 


1
2
3
4
5
6
7
8
9
10
11
12
13
14
15
16
17
18
19
20
21
22
23
24
25
26
27
28
29
30
31
32
33
34
35
36
37
38
39
40
41
42
43
44
45
46
47
48
49
50
51
52
53
54
55
56
57
58
59
60
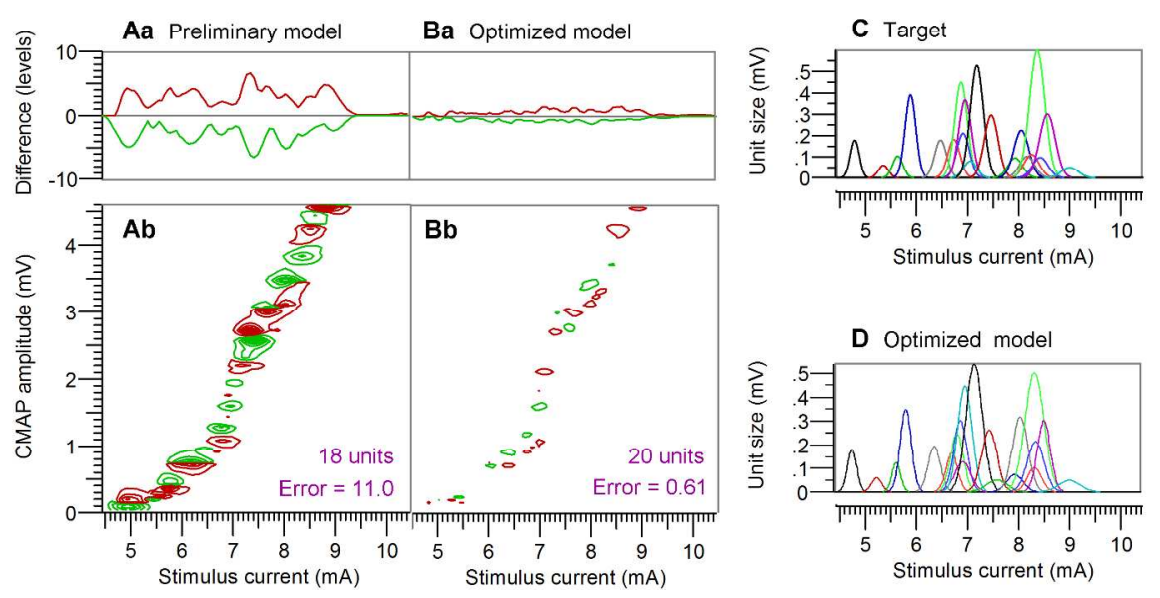

Figure 3

$254 \times 190 \mathrm{~mm}(300 \times 300$ DPI $)$

John Wiley \& Sons, Inc. 

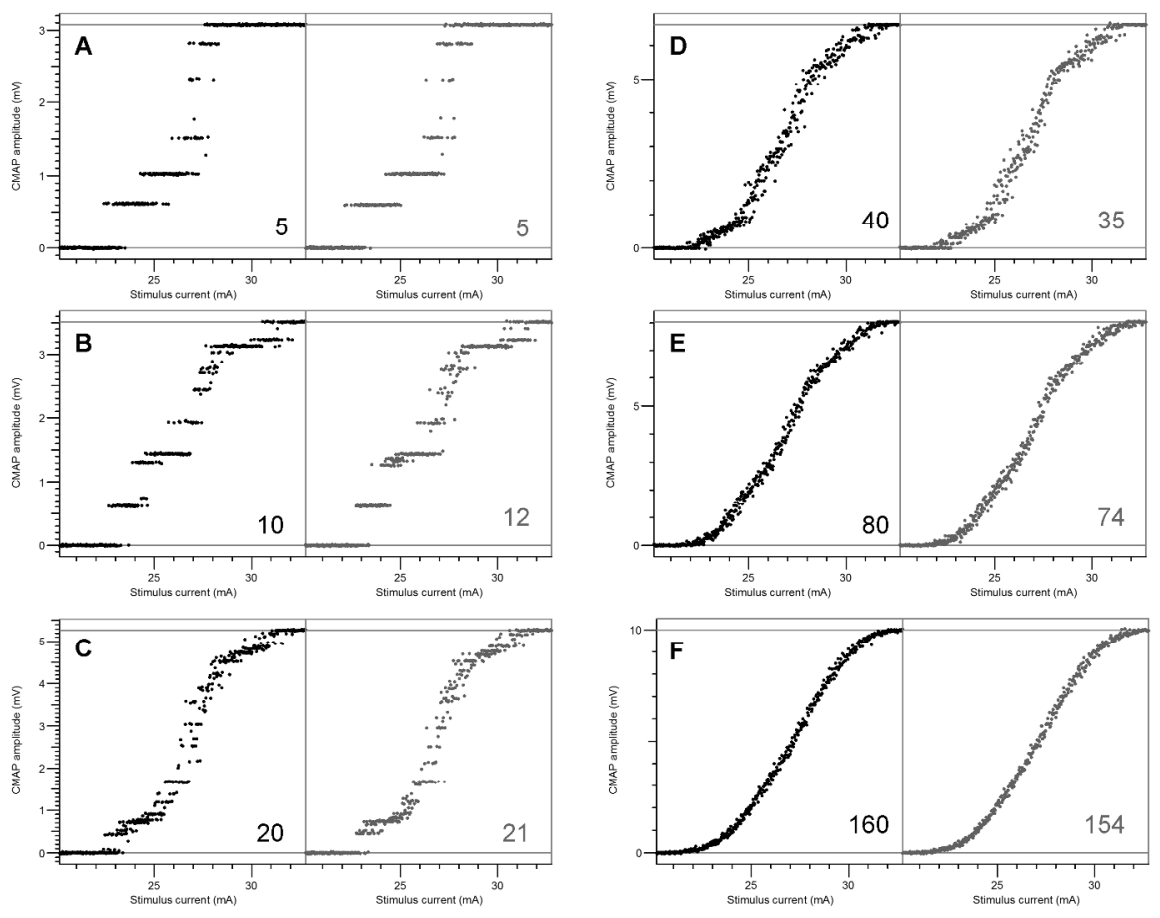

Figure 4

$254 \times 190 \mathrm{~mm}$ (300 x 300 DPI)

34

35

36

37

38

39

40

41

42

43

44

45

46

47

48

49

50

51

52

53

54

55

56

57

58

59

60

John Wiley \& Sons, Inc. 
Figure 5

$254 \times 190 \mathrm{~mm}$ (300 x 300 DPI)

John Wiley \& Sons, Inc. 